\title{
Electrophysiological evidence for the influence of unitization on the processes engaged during episodic retrieval: Enhancing familiarity based remembering
}

\author{
Sinéad M. Rhodes*, David I. Donaldson \\ The Psychological Imaging Laboratory, Department of Psychology, University of Stirling, Stirling FK9 4LA, UK \\ Received 18 January 2006; received in revised form 10 May 2006; accepted 2 June 2006
}

\begin{abstract}
Episodic memory depends upon multiple dissociable retrieval processes. Here we investigated the degree to which the processes engaged during successful retrieval are dependent on the properties of the representations that underlie memory for an event. Specifically we examined whether the individual elements of an event can, under some conditions, be unitized, leading to an enhancement of familiarity based responding. Retrieval processes were examined using event-related potential (ERPs) old/new effects, recorded during an associative recognition memory task. The nature of to-be-remembered information was manipulated by using word-pairs as stimuli. At study, participants were asked to remember wordpairs sharing an association (traffic-jam); association + semantic relationship (lemon-orange); or a semantic relationship only (cereal-bread). A behavioural pre-test revealed that association word-pairs were rated as having the most unitized representation. At test, participants were required to recognize if word-pairs were presented in the same pairing as at study, were rearranged from at study, or were entirely new. Behavioural recognition performance was clearly influenced by the nature of the to-be-remembered stimuli, memory being strongest for pairs related purely by association, and weakest for semantic only pairs. ERP old/new effects recorded at test also showed significant differences in the neural correlates of retrieval, depending on stimulus characteristics. The bilateral frontal old/new effect (typically associated with familiarity) was solely elicited by association only pairs. By contrast, the left parietal old/new effect (associated with recollection) was elicited equally by all three conditions. In addition, the late right frontal old/new effect (typically associated with some form of strategic/executive processing) was modulated. This latter effect was initially largest for association only pairs, and subsequently largest for semantic pairs. These findings suggest that the pattern of engagement of familiarity and recollection during successful episodic retrieval is dependent on the properties of the representations that underlie memory for an event.

(C) 2006 Published by Elsevier Ltd.
\end{abstract}

Keywords: ERPs; Episodic retrieval; Associative recognition; Unitization; Association; Semantic relationships

\section{Introduction}

Episodic memory supports the conscious retrieval of information about previously experienced events. The processes engaged during episodic memory retrieval are typically explained by dual process theories (e.g. Atkinson \& Joula, 1973; Jacoby, 1991; Mandler, 1980), whereby retrieval is thought to rely on two distinct processes: familiarity and recollection. Considerable evidence from behavioural studies suggests that familiarity is a relatively automatic process involving recognition without the retrieval of contextual information, while

\footnotetext{
* Corresponding author. Tel.: +44 1786 466849; fax: +44 1786467641.

E-mail address: s.m.rhodes@stir.ac.uk (S.M. Rhodes).
}

recollection is a more controlled process that supports retrieval of information and its context (Yonelinas, 2002). Whilst much is known about the characteristics of familiarity and recollection, less is known about the conditions that influence their engagement; why should familiarity rather than recollection be called upon to support retrieval? In the present study we provide one answer to this question; we employ event-related potentials (ERPs) to investigate the extent to which the processes engaged during successful retrieval are dependent on the properties of the representations that underlie memory for an event.

ERP studies have made an important contribution to the characterization of retrieval processes engaged during episodic memory task, providing support for dual process models of retrieval. The ERP old/new effect (the difference in activity 
between correctly recognized old and correctly rejected new stimuli) reflects the average pattern of activity that occurs during retrieval, time-locked to the onset of test stimuli, and reveals clearly identifiable neural correlates of familiarity and recollection. The bilateral frontal old/new effect, which has an early onset (300-500/600 ms) and is maximal over bilateral frontal electrodes, has been associated with familiarity. This effect is elicited by know responses (Klimesch et al., 2001), and to recognized items that are not associated with correct associative memory judgments (Curran, 2000). By contrast, a later onsetting old/new effect that is maximal over left parietal electrodes $(500-800 / 900 \mathrm{~ms})$ has been associated with recollection. The left parietal effect is elicited by remember responses (Klimesch et al., 2001; Trott, Friedman, Ritter, Fabiani, \& Snodgrass, 1999), by source memory tasks requiring the retrieval of contextual information (Wilding \& Rugg, 1996), and associative recognition (Donaldson \& Rugg, 1998, 1999).

While familiarity and recollection can be identified and dissociated during the retrieval of single items, the distinction between the processes is particularly clear when item recognition is compared with memory for associations. Associative recognition tasks involve the presentation of pairs of stimuli at study (e.g. dog-box, chair-rope, house-banana), and require discrimination between intact (e.g. dog-box) and rearranged (e.g. chair-banana) pairs at test. It is typically assumed that associative recognition tasks rely on recollection because they require the retrieval of an item and its context; because both intact and rearranged stimuli are familiar, successful performance requires recollection (cf. Yonelinas, 1997, 2002). ERP evidence is consistent with this view. As dual process theories predict, successful retrieval during associative recognition tasks that employ word-pairs as stimuli has been shown to reveal the left parietal index of recollection (e.g. Donaldson \& Rugg, 1998, 1999). Importantly, these ERP studies introduced new pairs at test, allowing the old/new effects to be compared using the same baseline of correctly rejected new stimuli as during tests of item memory. Although the two classes of old item are familiar compared to new items, successful discrimination between intact and rearranged pairs still requires recollection.

Implicit to the dual process account of associative recognition is the assumption that the individual elements of a stimulus are represented and processed separately. Here we challenge this assumption, and aim to demonstrate the influence of unitization on episodic retrieval. In normal use, episodic memory supports the retrieval of multi-faceted events; individual elements of an event may be recognized on the basis of being familiar, but retrieval of the relationship between the elements necessarily requires recollection (retrieval of context). This view of retrieval is based on assumptions about the way that information is represented within memory. According to this view, familiarity cannot support associative recognition tests because there is no specific representation of the relationship that can be associated with a sense of familiarity. The individual elements of the stimuli are represented separately, and can be familiar, but retrieval of the relationship requires recollection. The typical dual process view of associative recognition does not, however, account for the fact that the individual elements of an event can, under some con- ditions, be associated with a separate unitized representation of the relationships itself.

Our hypothesis is that if stimuli are unitized, associative recognition does not necessitate recollection, because the presence of a higher-level representation can give rise to a sense of familiarity. By this view the degree of engagement of familiarity and recollection during retrieval is likely to be dependent at least in part on the properties of the to-be-remembered episode. Behavioural evidence of recognition memory for faces provides some support for this argument. Yonelinas, Kroll, Dobbins, and Soltani (1999) reported that familiarity can support associative recognition for faces when face stimuli are presented upright, whereas this is not the case when faces are inverted. As face recognition is highly practiced and faces are normally processed holistically, the authors concluded that the upright faces were encoded as a coherent entity leading to a reliance on familiarity. The present study will investigate the influence of unitization on the engagement of episodic retrieval processes for word-pairs.

Before introducing the current experiment it is necessary to outline the types of relationships between words that may lead to a unitized representation. Relationships are often formed between words because they share a semantic relationship in common, reflecting activation of a semantic knowledge system in which information is organized categorically (e.g. violin-guitar). Neuropsychological studies provide compelling evidence that semantic knowledge is organized categorically. A recent review of 79 neuropsychological case studies concluded that there is strong evidence for category specific deficits for animate objects (animals), inanimate biological objects (fruits and vegetables), and artefacts (objects) (Capitani, Laiacona, Mahon, \& Caramazza, 2003). Evidence also suggests that functional relations (e.g. broom-floor) are an important part of this organized semantic system. For example, preserved access to functional properties of objects, despite impaired access to categorical knowledge, has been reported in a patient with semantic dementia (Moss, Tyler, Hodges, \& Patterson, 1995). Thus, the presence of pre-existing semantic relationships between stimuli is one possible way in which unitization could be encouraged.

In addition to forming relationships between items of information because of shared semantic properties, relationships can also be based purely on association, whereby one item calls to mind the other (e.g. traffic-jam). Such associations reflect word use rather than word meaning, and can exist either with (e.g. traffic-car) or without (e.g. traffic-jam) the presence of a semantic relationship. Studies of non-conscious priming provide evidence that relationships based on associative or semantic properties produce measurable changes in behaviour. For example, priming studies have revealed facilitated recognition, in the form of faster reaction times, for the presentation of a target word (e.g. dog) when it is preceded by an associatively (Ferrand \& New, 2003; Williams, 1996) or semantically (Moss, Ostrin, Tyler, \& Marslen-Wilson, 1995; Nation and Snowling, 1999) related word (e.g. cat) over an unrelated word (e.g. table). These studies typically aim to avoid conscious retrieval and consequently priming effects are thought to reflect automatic activation of the underlying representations of the concepts in semantic memory (Moss, Ostrin, et al., 1995). 
Further inspection of findings from priming studies, however, reveals the importance, and under some conditions the necessity, of association in eliciting automatic activation of concepts from semantic memory. For example, evidence that a semantic relationship is sufficient to elicit priming is restricted to the auditory domain (Moss, Ostrin, et al., 1995; Nation and Snowling, 1999). Priming experiments conducted in the visual domain reveal an additional association between the words is necessary to elicit a significant effect (Moss, Ostrin, et al., 1995; Shelton \& Martin, 1992). Furthermore, neuropsychological evidence from studies of non-conscious priming suggests dissociation between access of semantic and associative representations. Patients with Alzheimer's disease (Glosser \& Friedman, 1991) and children with poor reading comprehension (Nation and Snowling, 1999) show preserved non-conscious priming activations to words related by association, but show no priming effect for those sharing a semantic relationship alone. These findings highlight the importance of association in accessing representations from semantic memory and suggest that a pre-existing association between stimuli is a further possible way in which unitization could be encouraged.

In summary, the present study uses electrophysiological methods to investigate the influence of unitization on the engagement of episodic retrieval processes for word-pairs. We hypothesize that the neural correlates of familiarity and recollection should vary according to the nature of the relationships between items in a to-be-remembered word-pair. A behavioural rating paradigm is used to provide an assessment of the degree to which word-pairs related by associative and semantic properties are considered a single unit. ERP old/new effects will then be used to investigate if unitized pairs rely on familiarity, as reflected by the elicitation of the bilateral frontal effect during retrieval of these pairs. Specifically, we examine whether the existence of a unitized representation based on a pre-existing relationship between words differentially influences the processes engaged during episodic memory retrieval. Our aim is to describe the extent to which the processes engaged during successful retrieval are dependent on the properties of the representations that underlie memory for an event.

\section{Methods}

\subsection{Participants}

Thirty-two right-handed students participated in the experiment, paid at the rate of $£ 5$ per hour. Data from seven participants were discarded due to there being insufficient artifact-free trials in the critical response categories. The mean age of the remaining 25 subjects was 21.04 (range 18-31), 15 of whom were female. All participants had normal or corrected to normal vision. Informed consent was collected in line with Stirling University Ethics procedures.

\subsection{Stimuli}

The stimuli comprised 408 word-pairs selected from nouns, verbs, and adjectives (ranging from 3 to 9 letters in length) from the Kucera and Francis (1967) corpus. These stimuli included 306 related word-pairs reflecting three types of relationships between words: (i) 'association', words that are associated but do not share a semantic relationship of a categorical or functional nature (e.g. trafficjam), (ii) 'association + semantic', words that are associated and also share a
Table 1

Examples of word-pairs for each relationship type

\begin{tabular}{lll}
\hline Association & Association + semantic & Semantic \\
\hline Traffic-jam & Lemon-orange & Cereal-bread \\
Fountain-pen & Stool-chair & Violin-guitar \\
Mars-bar & Brother-sister & Cow-goat \\
Spark-plug & Lion-tiger & Prince-duke \\
Glow-worm & Gold-silver & Pig-chicken \\
Spare-tyre & Comb-brush & Sheep-rabbit \\
Grave-digger & Foam-rubber & Fork-plate \\
Dolly-bird & Needle-thread & Broom-floor
\end{tabular}

categorical or functional relationship (e.g. lemon-orange), and (iii) 'semantic', words sharing a categorical or functional relationship independent of association (e.g. cereal-bread). See Table 1 for examples. A further 102 pairs that did not share an associative or semantic relationship were added as fillers to prevent participants making recognition judgments based on whether the wordpairs made meaningful sense or not (see description of rearranged responses in procedure). Importantly, word-pairs of each relationship type were matched for word frequency (mean: 30.9) using the Francis and Kucera norms, and for both the presence and absence of association and semantic relationships (see Table 2).

Word-pairs in the association + semantic and semantic conditions were matched for semantic distance using a semantic space model; a method derived from the frequency distributions of the words occurring in the immediate context of a target word, computed over a large language corpus (containing millions of words) (McDonald, 2006). Association word-pairs shared a low contextual frequency of co-occurrence. Association ratings for word-pairs characterized by association and associative + semantic relationship were taken from the Edinburgh Association Thesarus (EAT, 2006) which gives the proportion of participants who called to mind the second word on presentation of the first (i.e. association rank). The EAT was chosen based on its established use in the literature (e.g. Coulson, Federmeier, Van Petten, \& Kutas, 2005) and because rank of association is regarded as a more optimal measure of association than association frequency (Anaki \& Henik, 2003). Semantic pairs had no associative relationship as indicated by this measure.

The degree to which word-pairs in each condition were considered to reflect a single unit was checked by carrying out a rating judgment pre-test. A behavioural rating paradigm was designed using E-Prime software (Psychology Software tools). Twenty participants, none of whom subsequently participated in the ERP experiment, were presented with word-pairs on a computer monitor. These wordpairs represented a randomly selected subset of the stimuli from the experiment ( 32 word-pairs in each condition). Participants were asked to "determine how unitized you think the word-pairs are, that is, how much you think these wordpairs would be considered as a single unit" using a scale ranging from 1 (little unitized) to 5 (completely unitized). Participants were told that there was no correct answer and that they were simply required to make a subjective judgment. Mean ratings for each condition of word-pairs are shown in Table 3. This pre-

Table 2

Data displayed relates to stimulus construction and memory performance

\begin{tabular}{lll}
\hline Relationship type & $\begin{array}{l}\text { Frequency of contextual } \\
\text { co-occurrence }\end{array}$ & Association strength \\
\hline Association & $0.178(0.007)$ & $0.206(0.01)$ \\
Association + semantic & $0.513(0.01)$ & $0.209(0.013)$ \\
Semantic & $0.523(0.007)$ & 0
\end{tabular}

Semantic distance ratings are a numerical representation of the meanings of words, derived from the frequency distributions of the words occurring in the immediate context of a target word, computed over a large language corpus (containing millions of words) (McDonald, 2006). Association strength refers to the proportion of participants who called to mind the second word as a first response on presentation of the first (Edinburgh Association Thesarus). Standard deviations are shown in brackets. 
Table 3

Data displayed relates to behavioural pre-test

\begin{tabular}{ll}
\hline Relationship type & Mean rating (S.D.) \\
\hline Association & $3.94(0.57)$ \\
Association + semantic & $3.75(0.74)$ \\
Semantic & $2.82(0.65)$
\end{tabular}

Mean ratings are shown of the degree to which word-pairs were rated as being associated with a single unit (range 1-5).

test revealed that word-pairs related purely by association are most considered to reflect a single unit.

\subsection{Procedure}

The experiment was designed using E-Prime software (Psychology Software tools) which allows precise stimulus timings. Word-pairs in uppercase 18-point courier new font were presented on a computer monitor. Letters were displayed in white font against a black background and were displayed one above the other slightly above and below central vision. At the viewing distance of $97 \mathrm{~cm}$, the stimuli subtended a maximum horizontal visual angle of approximately $3.7^{\circ}$, and a maximum vertical visual angle of approximately $1.4^{\circ}$. Responses were made on a Psychology Software Tools Serial Response box. Prior to commencing the task, each participant completed a practice session which included 8 words at study and 12 at test. The experiment was divided into 17 blocks of study and test. Study blocks comprised 16 word-pairs. Test blocks comprised 24 wordpairs, 8 of which were the same as presented at study, 8 of which were in a different pairing from study (rearranged), and 8 which were entirely new. Both study and test blocks involved equal proportions and randomized presentation of word-pairs across the relationship types. At study, participants were instructed to remember each word-pair as a pair.

In the study phase, each trial began with an initial fixation cross (+) displayed in the center of the screen for $1000 \mathrm{~ms}$. This cross was used to maintain participants' fixation on the center of the screen and to indicate the presentation of the next word-pair. A $1000 \mathrm{~ms}$ blank screen then preceded the presentation of the word-pair which was presented for $1500 \mathrm{~ms}$. Test phases immediately followed study phases. Each test trial began with a fixation cross which was presented for $1000 \mathrm{~ms}$ and which was followed by a blank screen also presented for $1000 \mathrm{~ms}$. Word-pair were presented for $2000 \mathrm{~ms}$ and followed by a $1500 \mathrm{~ms}$ blank screen. Participants had to make a response of same, rearranged, or new during the presentation interval. The end of this interval began a new trial. Participants make a response of same, rearranged, or new. Rearranged pairs were not of primary interest and ERPs for these pairs are not reported. However, their inclusion was important as the presence of rearranged pairs forces participants to respond old only when they remember the specific relationship between members of a word-pair, not just that both words in the pair are old. The process of rearranging related word-pairs results in the breaking up of the meaningful relationship between the words which introduces a potential confound. Participants could use their knowledge that a lack of meaningful relationship between the words indicates a word-pair is rearranged to decide whether pairs are rearranged or not, rather than remembering the specific relationship between the words. The inclusion of the rearranged condition in a study of related words thus necessitated the incorporation of filler words, which did not share an associative or semantic relationship when presented either in the same or in a rearranged pairing at test. Response hands for same and new were counterbalanced across participants.

\subsection{ERP recording}

Scalp EEG was recorded from 61 standard sites based on an extension of the international 10-20 system (Jasper, 1958): FZ, FCZ, CZ, CPZ, PZ, POZ, Oz, FP1, FP2, AF7, AF8, AF3, AF4, F7, F8, F5, F6, F3, F4, F1, F2, FT7, FT8, FC5, FC6, FC3, FC4, FC1, FC2, T7, T8, C5, C6, C3, C4, C1, C2, TP7, TP8, CP5, CP6, CP3, CP4, CP1, CP2, P7, P8, P5, P6, P3, P4, P1, P2, PO7, PO8, $\mathrm{PO} 5, \mathrm{PO} 6, \mathrm{PO} 3, \mathrm{PO} 4, \mathrm{O} 1, \mathrm{O} 2$. An additional EEG channel was recorded from the right mastoid. All channels were referenced to the left mastoid, and ERPs were algebraically reconstructed off-line to represent recordings with respect to an average mastoid reference. Vertical and horizontal EOG was recorded from bipolar pairs of electrodes placed above and below the left eye, and on the outer canthi. Inter-electrode impedance levels were kept below 5000. EEG and EOG were filtered with a bandpass of $0.01-40 \mathrm{~Hz}$ and digitized (16 bit) at a rate of $8 \mathrm{~ms}$ per point. Individual $1936 \mathrm{~ms}$ epochs were formed (beginning with a $104 \mathrm{~ms}$ pre-stimulus baseline) and epochs with baseline drift exceeding $75 \mu \mathrm{v}$, or base-to-peak amplitude exceeded $100 \mu \mathrm{v}$, were rejected. Averaged ERP waveforms were baseline corrected and smoothed over a 5-point kernel. A minimum of 16 artefact free trials in each critical response category was required from each participant to ensure an acceptable signal-to-noise ratio. The mean number of trials contributing to the grand average ERPs were: association: same (25) and new (26); association + semantic: same (24) and new (26); semantic: same (23) and new (27). Analysis was performed on mean voltage data relative to the pre-stimulus baseline period using repeated measures ANOVA, and only main effects or interactions involving the factors of condition (association, association + semantic, semantic) and/or response (same, new) are reported. The Geisser-Greenhouse correction for non-sphericity of data was applied as appropriate, and corrected d.f. and $F$ values are reported. All topographic analyses were performed on difference waveforms (same minus new responses) using rescaled data (McCarthy \& Wood, 1985).

\section{Results}

\subsection{Behavioural data}

As can be seen from Fig. 1a, superior recognition accuracy was observed for association word-pairs, driven largely by a greater hit rate for same pairs. A repeated measures ANOVA on hits (same and rearranged pairs) and correct rejections with factors of condition (association, association + semantic, semantic) and response (same, rearranged, new) revealed a main effect of condition $[F(2,48)=7.76, p=0.001]$, a main effect of response $[F(2,48)=6.31, p=0.004]$, and an interaction between condition and response $[F(4,96)=4.59, p=0.002]$ (see Fig. 1). Subsidiary ANOVAs revealed a significant effect of condition for same responses $[F(2,48)=8.54, p<0.001]$. Importantly, pairwise comparisons revealed increased hits for association than for either association + semantic $(p<0.01)$ or semantic wordpairs $(p<0.001)$. There was no significant difference in accuracy for same responses between association + semantic and semantic word-pairs $(p>0.05)$. ANOVA revealed a significant effect of condition for rearranged responses $[F(2,48)=3.71, p<0.04]$. Pairwise comparisons revealed increased hits for association $(p<0.02)$ and association + semantic $(p<0.05)$ than semantic word-pairs. There was no significant difference in accuracy between association and association + semantic word-pairs $(p>0.05)$. As expected, there were no significant differences in behavioural performance for new responses across relationship types.

As can be seen from Fig. 1b, word-pairs related by association and association + semantic elicited quicker response times across response types. A repeated measures ANOVA on reaction time data with factors of condition and response revealed a main effect of condition $[F(2,48)=10.63, p<0.001]$, a main effect of response $[F(1.4,32.9)=55.37, p<0.001]$, but no interaction between condition and response. Pairwise comparisons revealed slower reaction times for semantic than association $(p<0.001)$ and association + semantic $(p<0.02)$ across the three response types. These results show quicker response times to word-pairs which share an associative relationship. 


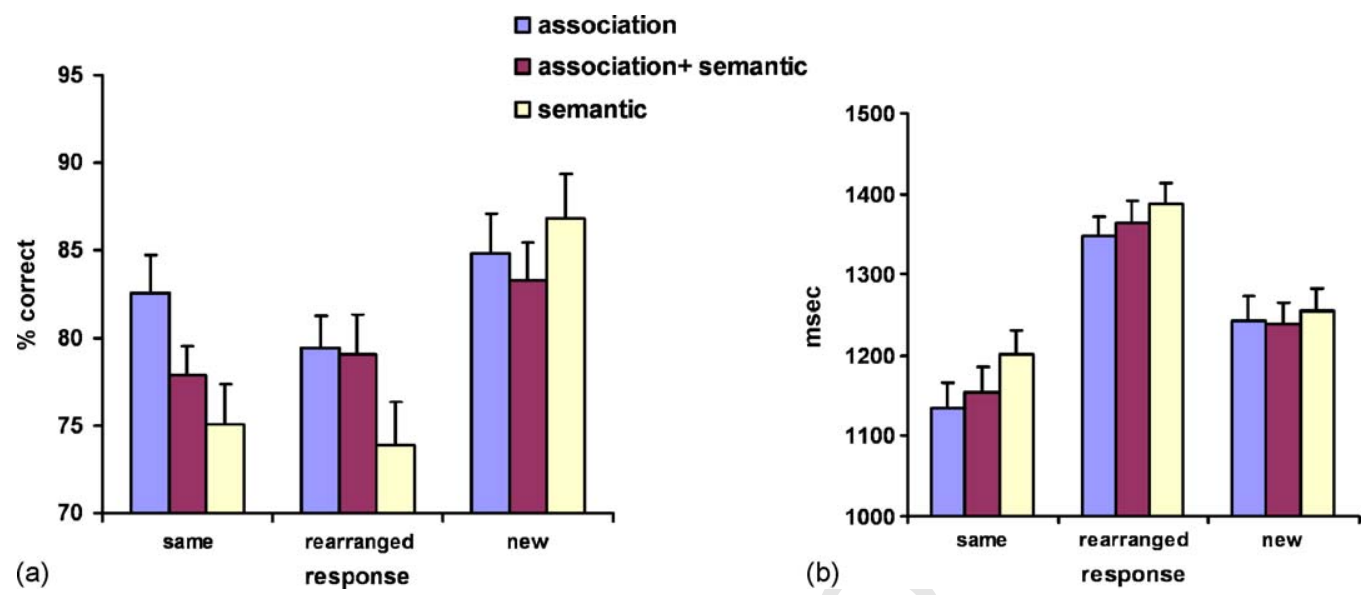

Fig. 1. Panel a: accuracy data, showing percentage of correct responses for each relationship type across three responses. Panel b: reaction time data, shown for the conditions in panel a. Error bars represent the standard error of the mean.

\subsection{ERP data}

The grand average ERPs elicited by associative word-pairs are shown in Fig. 2. The waveforms diverge about $250 \mathrm{~ms}$ poststimulus onset, with the ERPs for same responses becoming more positive than new responses, a difference that is maximal over frontal sites. This early frontal positivity is only evident for associative pairs, and the initial bilateral distribution becomes more right-sided from about $600 \mathrm{~ms}$. From about $800 \mathrm{~ms}$ this right-sided frontal positivity is also apparent for association + semantic and semantic word-pairs (see Figs. 3 and 4). From $1400 \mathrm{~ms}$ onwards the right-sided frontal positivity is maximal for semantic pairs, clearly extending until the end of the recording epoch (see Fig. 4). Positive activity is also observed over centro-parietal and parietal sites for same responses in comparison to new responses for all relationship types (see Figs. 2-4). This parietal positivity appears to emerge around $400 \mathrm{~ms}$, continuing until about $800 \mathrm{~ms}$ when the positive activity becomes maximal over right frontal sites. Based on visual inspection of the grand average waveforms, data was divided into time windows of $250-400$ and $400-750 \mathrm{~ms}$ for analyses relating to the bilateral frontal and left parietal effects, respectively. The presence of old/new differences post $800 \mathrm{~ms}$ which appear to show the time-course and distribution of the late right frontal effect led to analyses of two further time windows: 800-1400 and 1400-1900 ms.

\subsubsection{ERP analyses}

ANOVAs were conducted on chains of frontal and parietal electrodes for all time windows (see Fig. 5a for 250-400 and $400-750 \mathrm{~ms}$ time windows; Fig. 5b for $800-1400$ and $1400-1900 \mathrm{~ms}$ time windows). ANOVAs with factors of response (same, new), location (anterior, posterior) hemisphere (left, right) and site (superior, mid, inferior) were conducted separately on ERPs for association, association + semantic and semantic conditions in order to describe old/new effects for each of the relationship types. Significant effects of response or response interactions were followed up with subsidiary ANOVAs or paired $t$-tests where appropriate. Signifi- cant old/new differences within conditions were followed up with ANOVA conducted on difference waveforms (same minus new) employing factors of condition (association, association + semantic and semantic), location (anterior versus posterior), hemisphere (left versus right) and site (superior versus mid versus inferior) in order to compare the magnitude of the effects elicited by each relationship type. Topographic analyses were also conducted, employing the same factors, to investigate if the old/new differences elicited by each relationship types reflected activity from the same neural generators. Analyses of the 250-400 and 400-750 ms time windows report data from 24 participants as the loss of data from electrode $\mathrm{f} 1$ for one of the participants led to their removal from this particular analysis.

\subsection{2. $250-400 \mathrm{~ms}$}

As can be seen from Fig. 6a and b, analyses of this time window revealed an old/new effect maximal over bilateral frontal sites for association word-pairs alone. The initial ANOVA for association word-pairs revealed a significant response $\times$ location $\times$ site interaction $[F(1.1,25.8)=3.82$, $p=0.05]$. Subsidiary analyses of separate locations revealed a significant old/new difference for associative pairs at the anterior location only, with same waveforms more positive than new waveforms $[F(1,23)=12.79, p<0.001]$. Importantly, no significant interaction between response and hemisphere revealed that the old/new effect was characterized by a bilateral distribution (see Figs. 2 and 6b). No significant main effects of response or response interactions were found for association + semantic pairs (all $F<1.6$ ) or semantic pairs (all $F<1.2$ ), revealing no old/new differences for either of these types of pairs. This pattern of results suggests that word-pairs related purely by association elicited an old/new difference consistent with the distribution and timing of the bilateral old/new effect; whereas those sharing a semantic relationship did not.

\subsection{3. $400-750 \mathrm{~ms}$}

As can be seen from Fig. 7, analyses from the 400 to $750 \mathrm{~ms}$ time window revealed that all three relationship types elicited an old/new effect over centro-parietal sites consistent with the 


\section{Association Same}

\section{Association New}

FP1

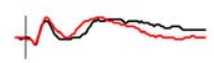

F7

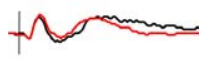

T7

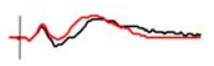

P7

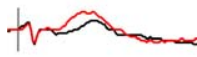

P07
F3

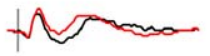

C3

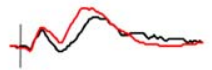

P3

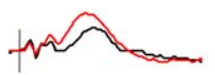

PO3

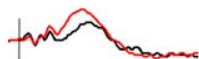

FZ

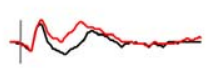

CZ

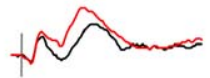

PZ

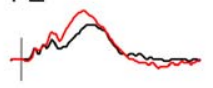

$\mathrm{OZ}$

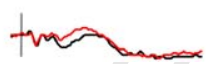

$+12$

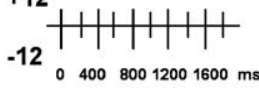

FP2
Nomanom

F4

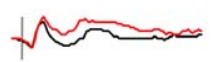

C4

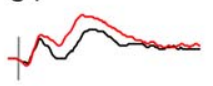

P4

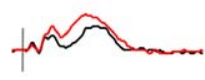

$\mathrm{PO} 4$

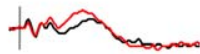

P8

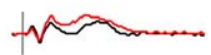

F8

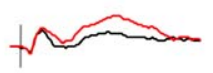

T8

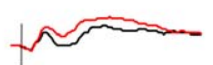

PO8

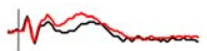

Fig. 2. ERPs for the recognized same and new responses for association word-pairs, showing from pre-stimulus to 1900 ms. Twenty-two electrodes are shown; across fronto-polar, frontal, central, parietal, and occipital scalp, arranged as if looking down onto the top of the head. Vertical bar shows stimulus onset (0 ms). Scale bar illustrates data is displayed positive up.

\section{Association + Semantic Same \\ Association + Semantic New}

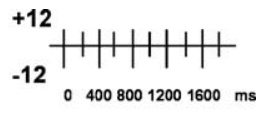

FP1

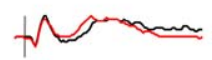

F7

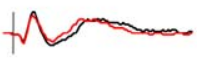

T7

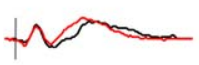

P7

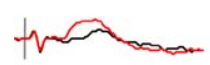

$\mathrm{PO} 7$

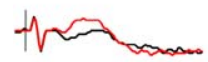

P3

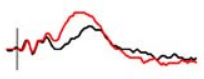

F3

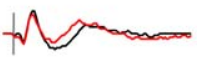

C3<smiles>C=CC=CCCC</smiles>

$\mathrm{PO} 3$

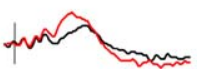

FZ

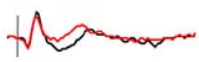

$\mathrm{CZ}$<smiles>CCCCCCCCC</smiles>

PZ<smiles>C=C=CCN=CC</smiles>

$\mathrm{OZ}$

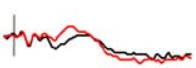

FP2

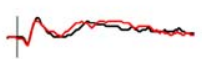

F4

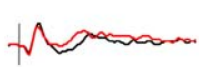

F8

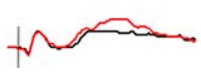

T8

C4

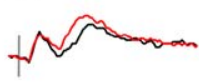<smiles>CCCCCCCC</smiles>

P8

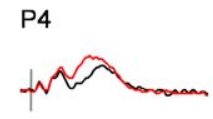

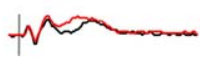

PO4

PO8

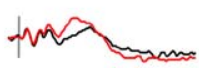

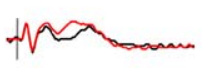

Fig. 3. ERPs for the recognized same and new responses for association + semantic word-pairs, showing from pre-stimulus to $1900 \mathrm{~ms}$. Twenty-two electrodes are shown; across fronto-polar, frontal, central, parietal, and occipital scalp, arranged as if looking down onto the top of the head. Vertical bar shows stimulus onset $(0 \mathrm{~ms})$. Scale bar illustrates data is displayed positive up. 


\section{Semantic Same}

Semantic New

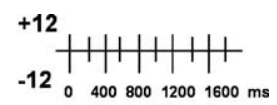

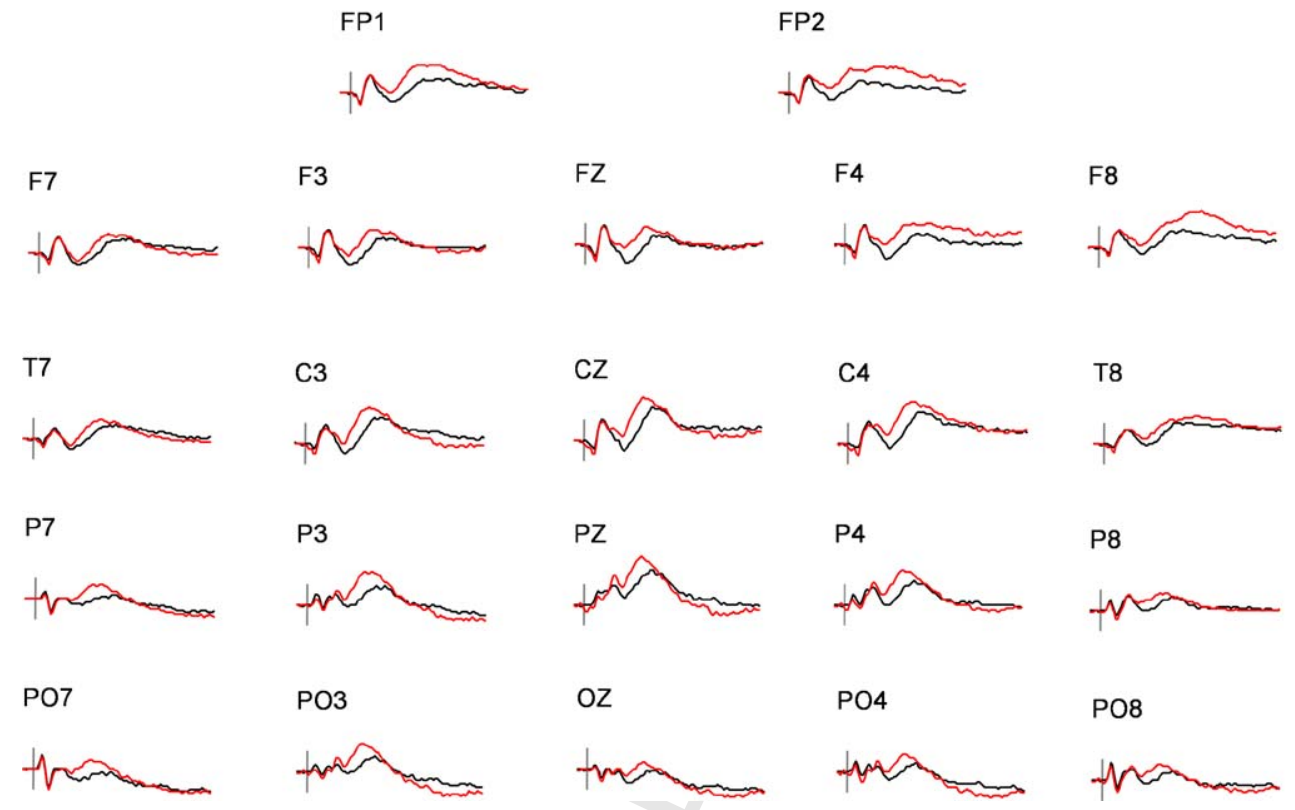

Fig. 4. ERPs for the recognized same and new responses for semantic word-pairs, showing from pre-stimulus to 1900 ms. Twenty-two electrodes are shown; across fronto-polar, frontal, central, parietal, and occipital scalp, arranged as if looking down onto the top of the head. Vertical bar shows stimulus onset (0 ms). Scale bar illustrates data is displayed positive up.

parietal effect associated with recollection as described in the literature. ANOVA revealed a significant old/new effect for association word-pairs. The initial ANOVA for association word-pairs revealed response $\times$ location $\times$ site $[F(1,23)=3.7, p=0.06]$, and response $\times$ site $[F(1.3,29.0)=5.66, p<0.02]$ interactions. Following the response $\times$ location $\times$ site interaction, subsidiary ANOVAs were conducted separately on anterior and posterior locations. ANOVA conducted on parietal sites revealed a main effect of response $[F(1,23)=5.06, p<0.04]$ revealing an old/new difference at parietal sites. Analysis of the anterior location revealed an interaction between response and site $[F(1.4,32.8)=3.61, p=0.05]$; paired $t$-tests on sepa- rate sites revealed a significant old/new difference at superior sites $(t=2.24, p<0.04)$. These analyses reveal old/new differences at both anterior and posterior locations for association word-pairs.

Analysis revealed a significant old/new effect for association + semantic word-pairs; the initial ANOVA revealed a response $\times$ location $\times$ site interaction $[F(2,46)=2.92, p=0.06]$. Subsidiary ANOVAs on separate locations revealed a condition $\times$ site interaction at parietal electrodes $[F(1.2,28)=0.07]$, which as can be seen from Figs. 3 and 7 reflects the greater positivity for same responses at superior sites. No significant effects of response were observed at the anterior location.

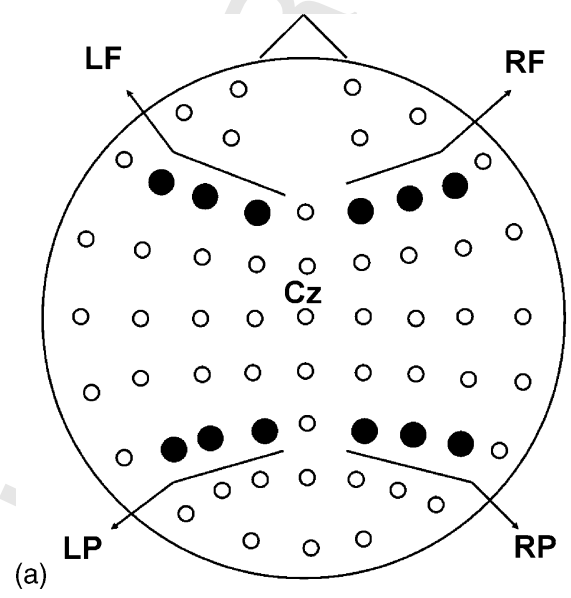

(b)

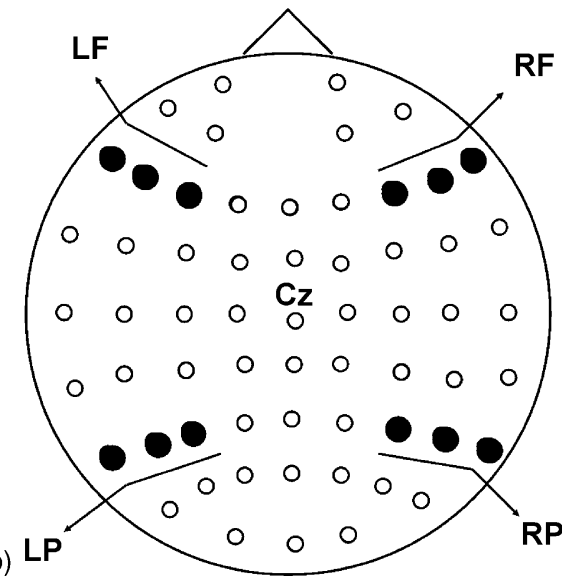

Fig. 5. Schematic maps of 61 electrodes sites with highlighted sites for each analysis. Panel a shows electrodes employed in the 250-400 and 400-750 ms time window, and panel b the 800-1400 and 1400-1900 ms time windows. LF: left frontal; RF: right frontal; LP: left parietal; RP: right parietal. 

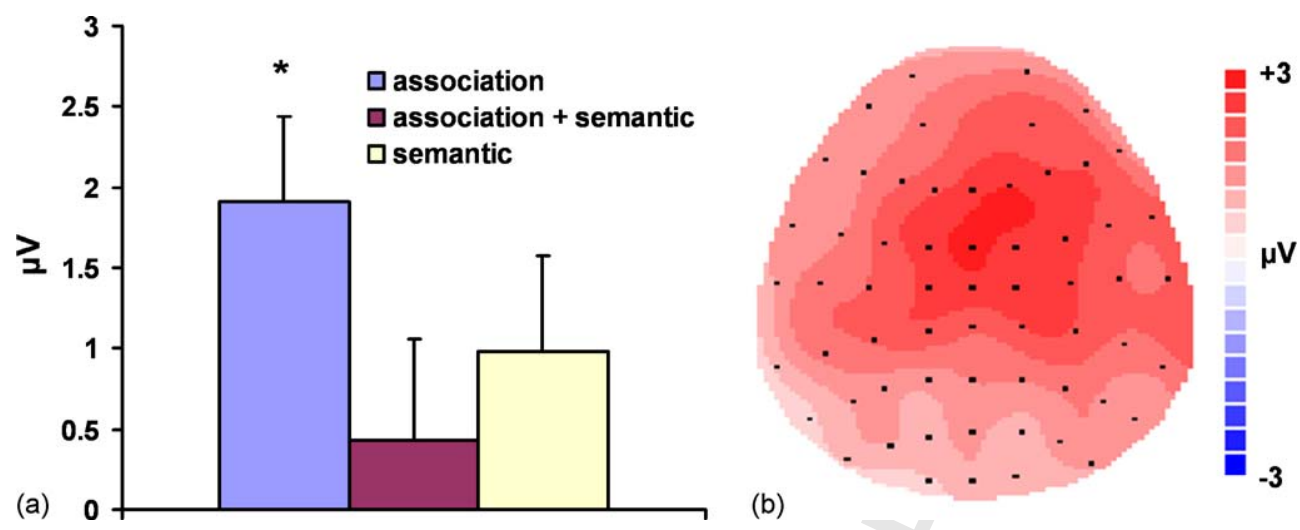

Fig. 6. The bilateral frontal effect elicited only for association pairs during the $250-400 \mathrm{~ms}$ time window. Panel a shows old/new effects for all three relationship types based on subtraction of hits to same pairs from correctly rejected new pairs collapsed across bilateral superior, mid, and inferior frontal electrodes, the asterisk indicates that there was a significant bilateral frontal old/new effect for the association word-pairs but not for the associative + semantic or semantic word-pairs. Error bars represent the standard error of the mean; panel b shows the topographic map illustrating the bilateral frontal old/new effect for association based on subtraction of hits to same pairs from correctly rejected new pairs. The scale bar to the right of the map indicates the maximum and minimum of the voltage range.

Analysis also revealed a significant old/new effect for semantic word-pairs. The initial ANOVA revealed a significant interaction between response and site $[F(2,46)=3.9, p<0.03]$. Paired $t$-tests conducted on superior, mid, and inferior sites collapsed across anterior and posterior locations revealed a significant old/new different at superior sites $(t=2.23, p=0.03)$.

Old/new effects elicited by the three relationship types over parietal electrodes were comparable in magnitude. A between condition analysis revealed interactions between condition, location, and site $[F(2.4,54.8)=2.68, p=0.06]$, and condition, location, and hemisphere $[F(2,46)=2.85, p=0.06]$. Subsidiary analyses revealed that the location interactions reflected significant differences between conditions at frontal sites; ANOVA revealed significant condition $\times$ hemisphere $[F(2,46)=3.47, \quad p<0.04]$ and condition $\times$ hemisphere $\times$ site $[F(4,92)=3.4, p<0.02]$ interactions at the anterior location, which as can be seen from Fig. 6 reflected a greater positivity for association than association + semantic at right hemisphere sites $(p<0.03)$, maximal at the right inferior site $(p<0.02)$. In contrast, no significant differences between relationship types were observed at the posterior location, demonstrating that the old/new effects elicited by each relationship type at parietal sites were similar in magnitude (see Fig. 7). Differences between relationship types was restricted to the anterior location; association alone produced a significant old/new effect at frontal sites and this most likely reflects residual spread of activity from the old/new effect observed for this condition in the earlier time window.

\subsection{4. $800-1400 \mathrm{~ms}$}

As can be seen from Figs. 2-4, analyses from the 800 to $1400 \mathrm{~ms}$ time window revealed that all three relationship types elicited an old/new effect over right frontal sites. A significant old/new effect was elicited by association wordpairs. ANOVA revealed an old/new effect with significant response $\times$ hemisphere and response $\times$ hemisphere $\times$ location interactions $[F(1,26)=6.14, p=0.02]$. As the location interaction was of most interest for describing the distribution of the old/new effect, this interaction was the focus for subsequent follow-up analysis. Subsidiary ANOVAs on separate locations revealed a significant old/new effect at frontal sites only, with a significant interaction between response and hemisphere $[F(1,24)=7.55, p<0.02]$, which paired $t$-tests revealed to reflect an old/new effect at right hemisphere sites $(t=2.67, p<0.03)$. A significant old/new effect was elicited by association + semantic word-pairs. ANOVA on association + semantic ERPs revealed

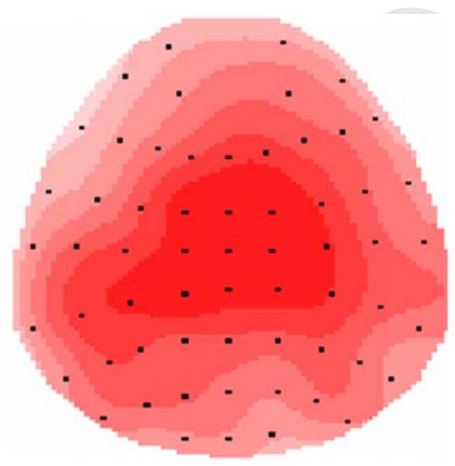

Association

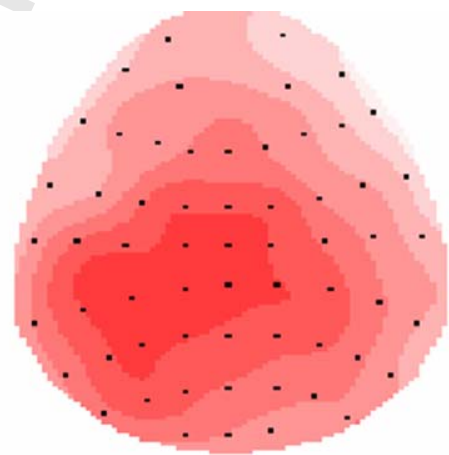

Association + Semantic
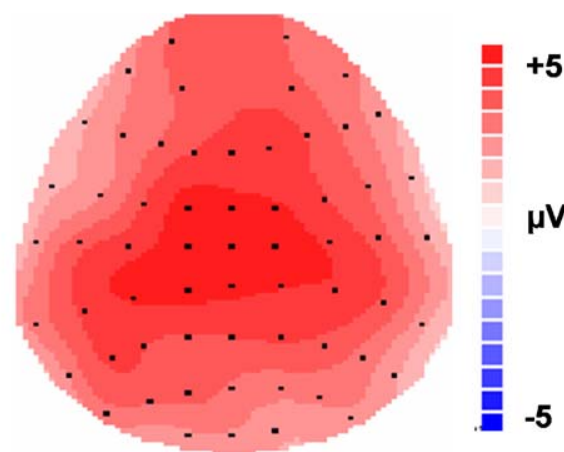

\section{Semantic}

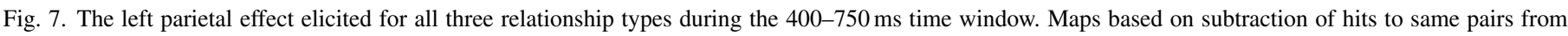
correctly rejected new pairs. The scale bar to the right of the map indicates the maximum and minimum of the voltage range. 
a response $\times$ hemisphere $[F(1,24)=3.85, p=0.06]$ interaction, which reflected a greater positivity for same pairs over right hemisphere sites. A significant old/new effect was elicited by semantic word-pairs. ANOVA on semantic ERPs revealed an old/new effect with significant response $\times$ location $[F(1,24)=5.97, p<0.03]$ and response $\times$ hemisphere $\times$ location $[F(1,24)=4.16, p=0.05]$ interactions. Subsidiary analysis on separate locations revealed that the significant interaction with location reflected an interaction between condition and hemisphere at frontal sites $[F(1,24)=4.14, p=0.05]$, which a paired $t$-test revealed to reflect an old/new effect at right hemisphere sites $(t=3.75, p<0.001)$. These analyses reveal significant old/new effects for each relationship type during 800 $1400 \mathrm{~ms}$.

A between condition analysis on difference waveforms revealed a significant three-way interaction between condition, hemisphere, and location $[F(2,48)=3.85, p<0.03]$. Subsidiary analyses on separate locations revealed a main effect of hemisphere at the anterior location $[F(1,24)=5.15, p<0.04]$. Paired $t$-tests for each relationship type revealed that the initial interaction reflected a significant difference between left and right hemisphere sites for association word-pairs, which as can be seen from Fig. 2 reflects a positivity for same responses at right hemisphere sites in contrast to negativity at left hemisphere sites. In summary, analysis of data during the $800-1400 \mathrm{~ms}$ time window reveal old/new effects for all three relationship types, consistent with the time-course and distribution of the late right frontal effect.

\subsection{5. $1400-1900 \mathrm{~ms}$}

As can be seen from Figs. 2-4, analyses from the 1400 to $1900 \mathrm{~ms}$ time window revealed that an old/new effect over right frontal sites was elicited for word-pairs related by either association or semantic but not for the combined association + semantic word-pairs. A significant old/new effect was elicited by association word-pairs; ANOVA revealed an old/new effect with a significant response $\times$ hemisphere $\times$ location interaction $[F(1,24)=4.97, \quad p<0.04]$. Subsidiary ANOVAs on separate locations revealed this interaction reflected a significant old/new difference at frontal sites alone. A significant response $\times$ hemisphere interaction $[F(1,24)=$ $6.48, p<0.02]$ followed up by paired $t$-tests revealed the interaction reflected the combination of positive activity at right hemisphere sites coupled with negative activity at left hemisphere sites $(t=-3.12, p<0.005)$. By contrast, no significant old/new effect was elicited by association + semantic word-pairs. ANOVA revealed no main effect of response or response interactions. However, a significant old/new effect was elicited by semantic word-pairs. ANOVA revealed significant response $\times$ location $[F(1,24)=4.89$, $p<0.04]$, response $\times$ hemisphere $[F(1,24)=8.87, p<0.007]$, response $\times$ location $\times$ hemisphere $[F(1,24)=9.32, p<0.005]$, and response $\times$ location $\times$ site $[F(1.4,34.1)=3.61, p=0.05]$ interactions. As description of the late right frontal effect is the key effect of interest, further analyses focused on following up the response $\times$ location $\times$ hemisphere interaction. Subsidiary analyses on separate locations revealed an old/new effect at frontal sites alone with a significant response $\times$ hemisphere interaction $[F(1,24)=10.68, p<0.003]$, which paired $t$-tests revealed to reflect a significant old/new effect at right frontal sites $(t=2.64, p<0.02)$.

A between condition analysis revealed a significant condition $\times$ location $\times$ hemisphere interaction $[F(2,48)=3.18$, $p=0.05]$. Subsidiary analyses conducted on separate locations revealed a significant interaction between relationship type and hemisphere at frontal sites $[F(2,48)=4.16, p<0.03]$, which paired $t$-tests revealed to reflect a combination of positive activity for same responses at right hemisphere sites and negative activity at left hemisphere sites for semantic pairs (see Fig. 4). Together these analyses reveal that post $1400 \mathrm{~ms}$ old $/$ new differences are only observed for the association and semantic conditions; this effect, consistent in distribution and timing with the late right frontal effect, is maximal for the semantic condition.

\subsubsection{Topographic analyses}

The first set of analyses was conducted to examine the neural generators of the old/new effects elicited in the 250-400 and $400-750 \mathrm{~ms}$ time windows. The first ANOVA was designed to examine changes in the early bilateral frontal effect and later parietal effect elicited by association word-pairs; a second ANOVA compared the topography of the parietal effect elicited by all three relationship types in the $400-750 \mathrm{~ms}$ time window. ANOVA conducted on association ERPs with factors of epoch (250-400 and 400-750 ms), location (anterior, posterior), hemisphere (left, right) and site (superior, mid, inferior) revealed an interaction between epoch and location $[F(1,24)=3.68, p=0.06]$. As can be seen from Figs. $6 \mathrm{~b}$ and 7 the epoch $\times$ location interaction reflected a shift from an anterior distribution of the old/new effect found for association wordpairs during $250-400 \mathrm{~ms}$ to a more posterior distribution during the $400-750 \mathrm{~ms}$ time window.

The second analysis compared relationship types during the $400-750 \mathrm{~ms}$ time window in order to ascertain whether the similar magnitude effects observed at the posterior location for each condition reflected activity from the same underlying neural generators. In each case, ANOVA with factors of condition (association, association + semantic, semantic), hemisphere (left, right) and site (superior, mid, inferior) on parietal sites (superior, mid, inferior) revealed no significant main effect of condition or condition interactions, suggesting no evidence for different neural generators underlying these parietal effects (see Fig. 7).

The next set of analyses was focused on examining the late right frontal effect elicited by the relationship types during the 800-1400 and 1400-1900 ms time windows. ANOVA with factors of condition (association, association + semantic, semantic), location (anterior, posterior), hemisphere (left, right), and site (superior, mid, inferior) conducted on data from the $800-1400 \mathrm{~ms}$ time window revealed no main effect of condition or condition interactions. This suggests that the effects elicited by association, association + semantic, and semantic during this time window reflected activity from the same neural generators. As only association and semantic pairs elicited an old/new difference during the 1400-1900 ms time window ANOVA was 
restricted to these two conditions and employed factors of condition (association, semantic), location (anterior, posterior), hemisphere (left, right), and site (superior, mid, inferior). Again this analysis revealed no differences between relationship types showing that the old/new effects elicited by association and semantic word-pairs did not differ in topographical distribution.

\section{Discussion}

The present ERP findings reveal striking dissociations between the influence of association, association + semantic relationships, and semantic relationships on episodic memory retrieval. These findings reveal that the processes supporting successful retrieval are differentially engaged according to the specific nature of the relationships between the elements of the to-be-remembered event. Association word-pairs were established at pre-test to be the most unitized of the three relationship types. The ERP findings revealed that a bilateral frontal old/new effect, typically associated with familiarity, was observed solely for word-pairs that shared only an association between them. The presence of an early bilateral frontal effect, coupled with superior recognition and faster reaction times for association wordpairs, suggests that the unitized representation associated with these words influenced the processes engaged during successful episodic retrieval by enhancing familiarity based remembering. This pattern of findings suggests that the individual elements of an event can, under some conditions, be sufficiently related that there is a separate unitized representation of the relationships itself. Below we address the influence of the nature of the relationship between stimuli on familiarity, and also discuss our findings relating to recollection, and the executive/strategic processes that support recognition.

Differential ERP effects between types of related pairs were observed at a time window and location associated with familiarity; a bilateral frontal old/new effect was solely observed for association pairs. Although familiarity is traditionally associated with item recognition, the present study suggests that familiarity was used to support retrieval on an associative recognition task. We interpret this finding as showing that prior experience of associations lead to the unitization of these word-pairs in memory. In line with behavioural studies which suggest superior recognition and faster reaction times in item than associative recognition, reflecting the characterization of familiarity as a fast automatic process (Yonelinas, 2002), we also found superior recognition and faster reaction times (albeit across responses) for association word-pairs. Thus, the behavioural evidence supports the ERP data in suggesting the relationship between association word-pairs facilitated the perception of a unit. The present findings contribute to dual process accounts of recognition memory, providing some insight into the operation of familiarity and recollection.

The engagement of familiarity in support of the retrieval of association word-pairs could be taken as evidence against the traditional view that familiarity can only be engaged in recognition of individual items and recollection is required for the retrieval of pairs of stimuli. By this view familiarity is available as a basis for recognition according to the extent that the pair is unitized. It is, however, unclear to what extent the associative stimuli should be considered pairs at all; they could be viewed as representing single items in memory, rather than pairs. From this perspective the current experiment is not a test of associative memory per se, and unitization should be viewed as little more than a novel means of manipulating item versus relational memory. This characterization ignores the specific nature of the task however, in particular the requirement to distinguish between same and rearranged pairs. Moreover, to our mind it is unclear whether the presence of an association necessarily prevents two words being processed as individual elements, or whether unitization is a context specific phenomenon. It would therefore be particularly interesting to discover whether the ERP correlates of familiarity are equally present for pairs rated as high and low unitized within a single associated condition. Nonetheless, our interpretation of the ERP findings is supported by the subjective behavioural ratings that revealed word-pairs related purely by association were most considered to reflect a single unit. Such subjective ratings must be treated with caution, and there is likely to be a relatively high degree of individual variability in such ratings. More importantly, such subjective feeling of unitization cannot be assumed to accurately reflect the nature of the underlying neural representations for these pairs.

The finding that the perception of a unit between items encourages familiarity based remembering has potential implications for the rehabilitation of cognitive impairment in patients who show impaired recollection in the face of preserved familiarity. Patients with the temporal lobe variant of fronto-temporal dementia, for example, show impaired recollection of source information but relatively good item recognition (Hodges \& Graham, 2001), and patients with Alzheimer's disease show impaired recollection in the face of preserved familiarity (Gallo, Sullivan, Daffner, Schacter, \& Budson, 2004). The present findings show that an associative relationship, independent of shared semantic properties, encourages the use of familiarity in conditions under which recollection is typically required. Similarly, patients with preserved familiarity could be trained to form new coherent entities by encouraging the formation of simple associations between what is otherwise perceived as random pairings of items. Such training could contribute to techniques designed to overcome the deficient recollection shown in these patients. The potential importance of association is also highlighted by the finding that patients with Alzheimer's disease show preserved non-conscious priming activations to words related by association (Glosser \& Friedman, 1991).

Our findings of a bilateral frontal effect elicited for association but not association + semantic word-pairs, conditions that were matched in degree of associative strength, inevitably leads to the question as to why the association + semantic pairs did not elicit this effect. While this pattern of results might seem surprising at first, behavioural data on the stimuli collected at pre-test revealed that words related purely by association were considered to reflect a single unit more than association + semantic pairs. Inspection of the pairs related by these two relationship types illustrates why only words related solely by association would be remembered as a unit. The key characteristic of the association word-pairs is the formation of a new coherent entity 
from words that previously had separate independent meaning (e.g. traffic-jam). In contrast, while association + semantic wordpairs are matched on associative strength, the words are still characterized entirely by their separate independent meanings (e.g. brother-sister). Thus, the new coherent entity arising from words with previously separate independent meanings in associative word-pairs appears to be important in the perception of a unit.

Whilst our findings point towards representations as critical for the pattern of processes engaged during associative retrieval, previous ERP studies have stressed the influence of the strategies employed by participants. For example, using unrelated wordpairs, Weyerts, Tendolkar, Smid, and Heinze (1997) manipulated relational processing by asking participants to either form a semantic association separately for each member of the pair, or to form a semantic association between the members of the pair. Only word-pairs that were semantically encoded elicited a parietal old/new effect. The authors conclude that relational encoding of word-pairs increased the reliance on recollection over familiarity. Similarly, Kounios et al. (2003) required participants to try and 'fuse' sequentially presented single words at study, and examined ERPs measured at retrieval according to whether this was possible or not. In this case no 'new' pairs were presented, thus traditional old/new effects could not be examined. The ERP findings were nonetheless interpreted as showing greater left parietal activity for fused than non-fused stimuli. In both of these studies, therefore, the suggestion is that changing participant strategies leads to differential processing of associated stimuli, and that this in turn leads to enhanced recollection based responding. The present experiments offer a different view, stressing the importance of the stimuli themselves, and perhaps as a result, pointing towards the possible role of familiarity for associative recognition performance.

Whilst we interpret our ERP findings in terms of the role of familiarity and recollection, other explanations for the observed ERP effects have been proposed. Although there is widespread consensus that the left parietal old/new effect reflects recollection, the claim that the bilateral frontal old/new effect reflects familiarity is more contentious. In particular, there is some evidence that early frontal old/new effects may in fact reflect conceptual priming rather than familiarity (e.g. Voss \& Paller, 2006; Yovel \& Paller, 2004). Our findings are, however, difficult to reconcile with a conceptual priming account. The present study reports a significant bilateral frontal old/new effect solely for the association condition. Whilst the existence of conceptual priming for purely semantic relationships remains under debate (cf. Hutchinson, 2003; Lucas, 2000) there is unequivocal evidence of priming effects for words sharing both associative and semantic relationships (Moss, Ostrin, et al., 1995; Shelton \& Martin, 1992). Thus, to our mind a conceptual processing interpretation would at the very least predict that a bilateral frontal old/new effect should be present for both of the associative conditions. Of course, the results from a single experiment cannot completely discount the conceptual priming account per se. Nonetheless, in the context of the wider literature, we reject the possibility that the early bilateral frontal old/new effects observed for associative pairs in the present experiment could reflect greater conceptual priming in this condition. ${ }^{1}$

More broadly, evidence suggests that conceptual priming effects require the repetition of both a stimulus and the processing required for that stimulus (Donaldson, Petersen, \& Buckner, 2001; Wagner, Koutstaal, Maril, Schacter, \& Buckner, 2000), which was not the case in the present experiment. To be clear, we do not believe that simple repetition of a stimulus at study and test during a memory experiment is necessarily sufficient to produce neural correlates of conceptual priming - even though the presentation of the stimulus will likely cause conceptual information associated with the stimulus to be activated on both occasions. By this view conceptual priming requires that the task demands associated with a stimulus be repeated (i.e. the repetition of the same information processing on the same stimulus representation). An important question remains as to whether neural measures can distinguish between the activation of the (conceptual) representations of a stimulus per se, and the (conceptual) processing associated with that representation.

In contrast to the selective elicitation of the bilateral frontal effect for association pairs, all three related word-pair types elicited a similar parietal effect associated with recollection. The presence of this parietal old/new effect is consistent with other studies that have employed associative recognition tasks, and has typically been associated with recollection (e.g. Trott, Friedman, Ritter, \& Fabiani, 1997). No differentiation occurred between the type of stimulus relationship and the parietal old/new effect, suggesting that retrieval of all three relationship types was supported by recollection. That is, the contribution of familiarity to recognition of association word-pairs did not appear to lead to a corresponding reduction in reliance on recollection. The present findings are thus consistent with the popular view that the engagement of familiarity and recollection are independent (e.g. Jacoby, 1991; Mandler, 1980). The behavioural evidence of facilitated recognition for association pairs supports this conclusion of the independent contributions of familiarity and recollection. A reliance on both familiarity and recollection, in contrast to semantic pairs (with and without association) which relied solely on recollection, provides a straightforward account of the improved recognition for associative pairs.

The presence of old/new effects from $800 \mathrm{~ms}$ post-stimulus that were maximal over the right frontal scalp led to analyses during two further time windows. Differential ERP old/new effects between types of related pairs were observed during these later time windows over right frontal electrodes. The late right frontal old/new effect (typically associated with some form of executive/strategic processing) was modulated by our manipulation of

\footnotetext{
1 Our interpretation is of course predicated on the philosophy that differences in the pattern of neural activity seen across conditions will mirror differences in the underlying processes engaged across conditions, and that this in turn will be mirrored by differences in behavioural measures of performance across conditions (where they exist). If differences in behavioural measures of a putative process do not cleanly mirror changes in a neural measure, we take this as evidence that the neural (and/or behavioural) measure simply does not provide an index of the putative process of interest.
} 
word-pairs. The effect was initially largest for association wordpairs, and subsequently largest for semantic pairs and absent for associative + semantic pairs. As noted above, this late onsetting right frontal ERP old/new effect has been associated with executive/strategic processes; most commonly referred to as search, monitoring, and evaluative processes, or as systematic or strategic operations. Such processes have traditionally been associated with prefrontal cortex (PFC) functioning (Fuster, 1989). The late right frontal effect is traditionally characterized as reflecting processes that act upon the products of retrieval (Wilding \& Rugg, 1996). The presence of the late right frontal effect in source memory (Wilding \& Rugg, 1996) and associative recognition tasks (Donaldson \& Rugg, 1998, 1999), and its association with remember responses (Trott et al., 1999) has led to the suggestion that it is typically contingent upon successful recollection (Donaldson \& Rugg, 1998). This view has, however, been challenged by demonstrations that the right frontal effect is elicited by false recognition in the absence of successful recollection (e.g. Curran, Schacter, Johnson, \& Spinks, 2001; Goldmann et al., 2003). Nonetheless even in these circumstances the effect is thought to reflect the engagement of effortful post-retrieval processes.

The present findings contribute to the functional characterization of the right-frontal old/new effect. The data are consistent with the characterization of this effect as reflecting executive/strategic processes and further suggest that the degree to which executive/strategic processes is dependent on the nature of the information to-be-retrieved, at least during successful episodic retrieval. The long lasting nature of the late right frontal effect for semantic word-pairs resembles those identified for unrelated word-pairs in previous studies (e.g. Donaldson \& Rugg, 1998, 1999). The behavioural data revealed reduced recognition for semantic pairs, and therefore the extension of the late right frontal effect post $1400 \mathrm{~ms}$ for these pairs is consistent with the interpretation that the late right frontal effect is associated with strategic processes required for the retrieval of information associated with more complex stimuli. Following this line of argument, however, it is unclear as to why the effect was absent for association + semantic pairs and not for association word-pairs post $1400 \mathrm{~ms}$. The behavioural data revealed increased recognition for association pairs in comparison to association + semantic pairs. This finding is therefore inconsistent with a sole explanation of the late right frontal effect as reflecting the retrieval of more complex stimuli. Further research is needed to clarify the precise cognitive operations associated with the late right frontal effect. Clearly the specific nature of the relationship between items is one factor in determining the elicitation of this effect. These findings therefore add to those reported for the differential influence of association and semantic relationships on familiarity, providing further evidence that the pattern of processes engaged during successful episodic retrieval is dependent on the nature of the information to-beretrieved.

The current study shows that the specific nature of the relationship between stimuli in associative recognition tasks influences the pattern of retrieval processes that are engaged. To date, most ERP studies of episodic retrieval have used simple stimuli, limiting the validity and generality of their findings. In the present study manipulation of the nature of the relationship between different elements of an episode reveals the differential influence of semantic knowledge and associations between items on successful retrieval. The findings suggest that the assumption that familiarity will not contribute to performance on associative recognition tasks is questionable if there is a unitized representation between the items. We support previous findings which have highlighted the role of association in influencing memory processing (Glosser and Friedman, 1999; Nation and Snowling, 1999), extending to the level of conscious episodic memory retrieval. In sum, these findings show that unitization has a significant influence on episodic memory retrieval; whether recollection or familiarity is engaged is ultimately dependent on the properties of the representation that underlie the information that is to-be-retrieved.

\section{Uncited references}

Collins and Loftus (1975), Herron and Rugg (2003), Hodgson (1991), Rugg, Schloerscheidt, Doyle, Cox, and Patching (1996), Thompson-Schill, Kurtz, and Gabrieli (1998) and Wilding and Rugg (1997a, 1997b).

\section{Acknowledgements}

This research was supported by the BBSRC. We would like to thank Catriona Bruce and Yvonne Archibald for their assistance in data collection.

\section{References}

Anaki, D., \& Henik, A. (2003). Is there a "strength effect" in automatic semantic priming? Memory \& Cognition, 31(2), 262-272.

Atkinson, R. C., \& Joula, J. F. (1973). Factors influencing speed and accuracy of word recognition. In S. Kornblum (Ed.), Proceedings of the fourth international symposium on attention and performance (pp. 583-611). New York: Academic Press.

Capitani, E., Laiacona, M., Mahon, B., \& Caramazza, A. (2003). What are the facts of semantic category-specific deficits? A critical review of the clinical evidence. Cognitive Neuropsychology, 20, 213-261.

Collins, A. M., \& Loftus, E. F. (1975). A spreading activation theory of semantic processing. Psychological Review, 82, 407-428.

Coulson, S., Federmeier, K. D., Van Petten, C., \& Kutas, M. (2005). Right hemisphere sensitivity to word and sentence level context: Evidence from event related brain potentials. Journal of Experimental Psychology: Learning Memory and Cognition, 31(1), 129-147.

Curran, T. (2000). Brain potentials of recollection and familiarity. Memory \& Cognition, 28, 923-938.

Curran, T., Schacter, D. L., Johnson, M. K., \& Spinks, R. (2001). Brain potentials reflect behavioral differences in true and false recognition. Journal of Cognitive Neuroscience, 13(2), 201-216.

Donaldson, D. I., Petersen, S. E., \& Buckner, R. L. (2001). Dissociating memory retrieval processes using fMRI: Evidence that priming does not support recognition memory. Neuron, 31, 1-20.

Donaldson, D. I., \& Rugg, M. D. (1998). Recognition memory for new associations: Electrophysiological evidence for the role of recollection. Neuropsychologia, 36, 377-395.

Donaldson, D. I., \& Rugg, M. D. (1999). Event-related potential studies of associative recognition and recall: Electrophysiological evidence for context dependent retrieval processes. Cognitive Brain Research, 8, 1-16. 
Edinburgh Association Thesarus. (2006). MRC psycholinguistic database, http://www.eat.rl.ac.uk/.

Ferrand, L., \& New, B. (2003). Semantic and associative priming in the mental lexicon. In P. Bonin (Ed.), Mental lexicon: Some words to talk about words (pp. 25-43). Hauppauge, NY: Nova Science Publisher.

Fuster, J. M. (1989). The prefrontal cortex: Anatomy, physiology and neuropsychology of the frontal lobe (2nd ed.). New York: Raven Press.

Gallo, D. A., Sullivan, A. L., Daffner, K. R., Schacter, D. L., \& Budson, A. E. (2004). Associative recognition in Alzheimer's disease: Evidence for impaired recall-to-reject. Neuropsychology, 18(3), 556-563.

Glosser, G., \& Friedman, E. B. (1991). Lexical but not semantic priming in Alzheimer's disease. Psychology and Aging, 6, 522-527.

Goldmann, R. E., Sullivan, A. L., Droller, D. B., Rugg, M. D., Curran, T., Holcomb, P. J., et al. (2003). Late frontal brain potentials distinguish true and false recognition. Neuroreport, 14(13), 1717-1720.

Herron, J. E., \& Rugg, M. D. (2003). Retrieval orientation and the control of recollection. Journal of Cognitive Neuroscience, 15(6), 843-854.

Hodges, J. R., \& Graham, K. S. (2001). Episodic memory: Insights from semantic dementia. Philosophical Transactions of the Royal Society of London: Series B, Biological Sciences, 356, 1423-1434.

Hodgson, J. M. (1991). Informational constraints on pre-lexical priming? Language and Cognitive Processes, 6, 169-205.

Hutchinson, K. A. (2003). Is semantic priming due to association strength or feature overlap? A microanalytic review. Psychonomic Bulletin and Review, 10(4), 785-813.

Jacoby, L. L. (1991). A process dissociation framework: Separating automatic from intentional uses of memory. Journal of Memory and Language, 30(5), 513-541.

Klimesch, W., Doppelmayr, A., Yonelinas, A. O., Kroll, N. E. A., Lazara, M., Rohm, D., et al. (2001). Theta synchronization during episodic retrieval: Neural correlates of conscious awareness. Cognitive Brain Research, 12(1), 33-38.

Kounios, J., Bachman, P., Casasanto, D., Grossman, M., Smith, R. W., \& Yang, W. (2003). Novel concepts mediate word retrieval from human episodic associative memory: Evidence from event-related potentials. Neuroscience Letters, 345, 157-160.

Kucera, H., \& Francis, W. N. (1967). Computational analysis of present-day American English. Providence, RI: Brown University Press.

Lucas, M. (2000). Semantic priming without association: A meta-analytic review. Psychonomic Bulletin and Review, 7, 618-630.

McCarthy, G., \& Wood, C. C. (1985). Scalp distributions of event-related potentials: An ambiguity associated with analysis of variance models. Electroencephalography and Clinical Neurophysiology, 62, 203-208.

McDonald, S. (2006). http://www.iccs.inf.ed.ac.uk/ smcdonal/semantic_ space_model.html.

Moss, H. E., Ostrin, R. K., Tyler, L. K., \& Marslen-Wilson, W. D. (1995). Accessing different types of lexical semantic information: Evidence from priming. Journal of Experimental Psychology: Learning, Memory and Cognition, 21(4), 863-883
Moss, H. E., Tyler, L. K., Hodges, J. R., \& Patterson, K. (1995). Exploring the loss of semantic memory in semantic dementia: Evidence from a primed monitoring study. Neuropsychology, 9(1), 16-26.

Rugg, M. D., Schloerscheidt, A. M., Doyle, M. C., Cox, C. J. C., \& Patching, G. R. (1996). Event-related potentials and the recollection of associative information. Cognitive Brain Research, 4(4), 297-304.

Shelton, J. R., \& Martin, R. C. (1992). How semantic is automatic semantic priming? Journal of Experimental Psychology: Learning, Memory and Cognition, 18, 1191-1210.

Thompson-Schill, S. L., Kurtz, K. J., \& Gabrieli, D. (1998). Effects of semantic and associative relatedness on automatic priming. Journal of Memory and Language, 38, 440-458.

Trott, C. T., Friedman, D., Ritter, W., \& Fabiani, M. (1997). Item and source memory: Differential age effects revealed by event-related potentials. $\mathrm{Neu}$ roreport, 8, 3373-3378.

Trott, C. T., Friedman, D., Ritter, W., Fabiani, M., \& Snodgrass, J. G. (1999). Episodic priming and memory for temporal source: Event-related potentials reveal age-related differences in prefrontal functioning. Psychology and Aging, 14(3), 390-413.

Voss, J. L., \& Paller, K. A. (2006). Fluent conceptual processing and explicit memory for faces are electrophysiologically distinct. The Journal of Neuroscience, 26(3), 923-926.

Wagner, A. D., Koutstaal, W., Maril, A., Schacter, D. L., \& Buckner, R. L. (2000). Task-specific repetition priming in left inferior prefrontal cortex. Cerebral Cortex, 10, 1176-1184.

Weyerts, H., Tendolkar, I., Smid, H. G. O. M., \& Heinze, H. J. (1997). ERPs to encoding and recognition in two different inter-item association tasks. Neuroreport, 8, 1583-1588.

Wilding, E. L., \& Rugg, M. D. (1996). An event-related potential study of recognition memory with and without retrieval of source. Brain, 119, 889-905.

Wilding, E. L., \& Rugg, M. D. (1997a). An event related potential study of memory for words spoken aloud or heard. Neuropsychologia, 35, 1185-1195.

Wilding, E. L., \& Rugg, M. D. (1997b). Event related potentials and the recognition memory exclusion task. Neuropsychologia, 35, 119-128.

Williams, J. N. (1996). Is automatic priming semantic? European Journal of Cognitive Psychology, 22, 139-151.

Yonelinas, A. P. (1997). Recognition memory ROCs for item and associative information: The contribution of recollection and familiarity. Memory \& Cognition, 25(6), 747-763.

Yonelinas, A. P. (2002). The nature of recollection and familiarity: A review of 30 years of research. Journal of Memory and Language, 46, 441-517.

Yonelinas, A. P., Kroll, N. E. A., Dobbins, I. G., \& Soltani, M. (1999). Recognition memory for faces: When familiarity supports associative recognition judgments. Psychonomic Bulletin and Review, 6(4), 654-661.

Yovel, G., \& Paller, K. A. (2004). The neural basis of the butcher-on-thebus phenomenon: When a face seems familiar but is not remembered. Neuroimage $R C, 21,789-800$ 